\title{
Estructura de la madera de Persea americana var. guatemalensis Mill (Hass)
}

\author{
José Antonio Silva Guzmán ${ }^{1}$ \\ Francisco Javier Fuentes Talavera ${ }^{1}$ \\ Hans G. Richter ${ }^{2}$ \\ Guillermo Angeles Alvarez ${ }^{3}$ \\ Rubén Sanjuan Dueñas ${ }^{1}$
}

\begin{abstract}
RESUMEN
En el presente trabajo se dan a conocer los resultados de un estudio de las características macroscópicas y microscópicas de la madera de Persea americana var. guatemalensis, Mill (Hass) procedente de plantaciones comerciales (huertas), motivado principalmente por la situación que prevalece en torno al cultivo y producción de aguacate en México y por ende, a la disponibilidad de esta madera, que es actualmente desaprovechada. Este trabajo forma parte de un estudio integral sobre las propiedades tecnológicas de la madera de esta especie. En función de las relaciones morfológicas de sus fibras, se hace un análisis tendiente a visualizar las características y propiedades de pulpas celulósicas resultantes, ante un eventual aprovechamiento para la producción de celulosa para papel. Se incluye información general sobre la distribución geográfica, taxonomía y reportes sobre otros estudios y aprovechamiento de este recurso forestal.
\end{abstract}

PALABRAS CLAVE:

Persea americana, aguacate, estructura y calidad de la madera, anatomía de la madera, celulosa y papel, usos.

\begin{abstract}
This paper presents the results of a study of the macroscopics and microscopic characteristics of the wood of Persea americana var. guatemalensis Mill (Hass). Samples were collected from commercial plantations. The study was motivated mainly by the situation prevailing around Mexican avocado production, and because this wood is considered to be underused. This study is part of a larger study on the technological characteristics of the wood of this species. From the fiber morphological relationships, an analysis is made about its characteristics as raw material for pulp and paper production. General information is included on the geographical distribution, taxonomy and reports on other studies and uses of this forest resource.
\end{abstract}

\section{KEY WORDS:}

Persea americana, avocado, wood structure and quality wood anatomy, pulp and paper, uses.

$1 \quad$ Universidad de Guadalajara, Departamento de Madera, Celulosa y Papel “Ing. Karl Augustin Grellmann”. Apdo. Postal 52-93, Zapopan, Jalisco C.P. 45020. México.

2 Bundesforschungsanstalt fur Forst-und Holzwirschaft. Leuschenerstrasse 91, 21031 Hamburgo, Alemania.

3 Instituto de Ecología, A.C., Depto. Productos Forestales y Conservación de Bosques. Apdo. Postal 63. Xalapa Ver. 91000 México.

Manuscrito recibido para su publicación el 14 de Enero de 1999. 


\section{INTRODUCCION}

Debido a su importancia económica, el cultivo del aguacate (Persea americana var. guatemalensis Mill) en México ha crecido en forma significativa en los años recientes; sin embargo, este cultivo sólo es masivo en algunos estados del país, principalmente Michoacán, que cuenta con una superficie plantada de casi 83000 ha, que producen 650,000 toneladas anuales, representando el $87 \%$ de la producción del país y el $44 \%$ de la producción total mundial (Quintero, 1998).

Con la apertura del mercado de los Estados Unidos de Norteamérica a la importación de aguacate mexicano, se ha generado un mayor interés por el establecimiento y aprovechamiento de este recurso agrícola. Sin embargo, en la actualidad la mayoría de las plantaciones presentan un problema generalizado ocasionado por una falta de planeación adecuada durante su establecimiento. Las distancias utilizadas entre árboles no son las óptimas, lo que ha generado una gran cantidad de biomasa proveniente de ramas y troncos, la cual tiene que ser forzosamente removida mediante podas y clareos de los árboles, con el propósito de mantenerlos en óptimas condiciones de producción. De no realizarse estas actividades, se genera una merma muy considerable en el rendimiento de la fruta y graves problemas fitosanitarios.

De acuerdo con comunicaciones de propietarios de plantaciones, se estima que en el aclareo se derriba de un 33 a 50\% de los árboles. El manejo de esta biomasa representa un gran problema para los propietarios de huertas. Generalmente, después de ser cortados los troncos y ramas, son quemados en las parcelas 0 utilizados como leña, sin obtenerse ningún beneficio económico de esta materia prima.

El aguacate (Persea americana Mill) pertenece a la familia Lauraceae, que cuenta con casi 2500 especies que crecen principalmente en los bosques nublados de montaña en zonas tropicales y subtropicales, especialmente en América y Asia. El género tiene casi 200 especies y se divide en tres subgéneros: Persea, Eriodaphne y Machilus. El número de especies del subgénero Persea difiere, de acuerdo a la clasificación seguida, entre cuatro y siete. Estas especies se distribuyen principalmente desde la parte central de México al extremo noreste de Sudamérica, en alturas desde el nivel del mar hasta 3500 msnm.

El aguacate es un árbol de $10-15 \mathrm{~m}$ de altura (cuando es cultivado) y alcanza diámetros de $40-60 \mathrm{~cm}$. En estado silvestre puede llegar a 25 ó $30 \mathrm{~m}$ de altura. Es probablemente originario de México y Centroamérica (Record y Hess, 1943; Kopp, 1966). Actualmente es cultivado en varias partes del mundo, por ejemplo en los Estados de California y Florida de los Estados Unidos de Norteamérica, y en otros países como Israel, España, Nueva Zelanda y Sudáfrica (Smith et al., 1992).

En México, el aguacate denominado comercialmente como "Hass" se cultiva principalmente en el estado de Michoacán y en menor proporción en Jalisco, Nayarit, Estado de México, Puebla y Veracruz (Smith et al., 1992). Los horticultores reconocen tres variedades con caracteres claramente diferenciables: antillana, guatemalensis y mexicana. La mayor parte de la investigación científica, está dirigida a estas variedades y sus relaciones filogenéticas. De acuerdo con varias hipótesis se señala a la zona maya como el área de origen y domesticación de la especie (Gama-Campillo, 1998). Estudios realizados por esta autora demostraron que los aguacates cultivados pertenecen principalmente a una misma especie, y que toda la variabilidad que se presenta se debe a efectos ecológicos, generados mediante un lento proceso de domesticación por la selección que el hombre ha hecho para su cultivo. Gama-Campillo propone tres nuevas combinaciones: $P$. americana var. 
guatemalensis (L. Wms.) Bergh ex Gama, $P$. americana var. floccosa (Mez) Bergh \& Scora ex Gama, y $P$. americana var. steyermarkii (Allen) Bergh \& Scora ex Gama (Gama-Campillo, 1994).

Respecto a las propiedades tecnológicas de la madera de aguacate, Rogel (1982) reporta un estudio sobre las características anatómicas de la madera de Persea americana y señala algunos usos potenciales de la madera. Tamarit (1996) la incluye dentro de la evaluación de índices de calidad de pulpa de 132 especies de madera, clasificándola de acuerdo a la relación de Runkel como excelente. Por su parte, Martínez-Pinillos y Martínez (1996) también la incluyen en su estudio de comportamiento al cepillado y lijado de 33 especies, reportando su comportamiento como excelente al cepillado con un ángulo de navajas de $30^{\circ}$, regular a $25^{\circ}$ y bueno a $20^{\circ}$. Su comportamiento al lijado lo califican como excelente con lija de grano 100 y muy pobre para los granos de 80 y 60 . Fuentes et al. (1998) presentan un estudio sobre el comportamiento higroscópico, calificándola como moderadamente estable de acuerdo con la anisotropía de la contracción y de una densidad anhidra promedio de 0.527 $\mathrm{g} / \mathrm{cm}^{3}$.

Del aprovechamiento de la madera de aguacate existen pocas referencias en nuestro país. Pennington y Sarukhán (1998) señalan aplicaciones en construcciones ligeras y objetos torneados; sin embargo, se trata de usos muy locales y no generalizados. Rogel (1982) también señala usos locales para tablas, leña, artículos torneados y costillas de guitarras comerciales. En el ámbito internacional, Chudnoff (1984) hace mención también a la aplicación en construcciones ligeras, pisos, muebles y carpintería en general e incluso en contrachapados, pero tampoco son usos muy extendidos.

Dado el volumen de biomasa de aguacate producido en el país, su aprovechamiento representa sin duda un especial interés para ser estudiado, con el propósito de incorporar nuevas materias primas a diversos procesos industriales 0 artesanales. El estudio de la estructura de su madera resulta de importancia primaria como indicador en su comportamiento tecnológico.

\section{OBJETIVOS}

Uno de los objetivos del presente trabajo es dar a conocer la estructura y calidad de la madera de aguacate cultivado (Persea americana var. guatemalensis Mill) a través de la descripción de sus características macroscópicas y la medición de sus principales elementos estructurales.

Con base en las características previamente mencionadas $y$ en la determinación de los coeficientes de Runkel, rigidez, flexibilidad e índice de esbeltez, se analiza la posibilidad de utilizar la madera de esta especie como materia prima para la producción de celulosa para papel. Adicionalmente, se busca promover el conocimiento de recursos maderables alternos, con lo cual se pueda motivar el interés de un aprovechamiento acorde a la compatibilidad de las propiedades del material y los requerimientos de los productos a elaborar.

\section{METODOLOGIA}

La madera utilizada en este trabajo fue colectada de una plantación de Persea americana var. guatemalensis de la población de Jujucato, Michoacán (Municipio de Santa Clara del Cobre) con árboles de 17 años de edad y aproximadamente $12 \mathrm{~m}$ de altura. Es importante señalar que los fustes de aguacate provenientes de huertas no son rectos, sino que generalmente se ramifican a muy corta distancia del suelo, regularmente antes de la altura de pecho, por lo que el mayor volumen de material 
utilizado en el presente estudio corresponde a ramas.

Para el presente trabajo se colectaron 10 trozas de $1 \mathrm{~m}$ de largo y $25 \mathrm{~cm}$ de diámetro procedentes de árboles que fueron derribados durante maniobras de aclareo. De cada una se elaboraron 4 tablillas para realizar las descripciones macroscópicas, las cuales fueron realizadas tomando como base la lista de caracteres microscópicos para la identificación de maderas duras publicada por la Asociación Internacional de Anatomistas de la Madera (IAWA, 1989) y utilizando para su observación una lupa de 10 aumentos.

La densidad de la madera de Persea americana fue determinada de acuerdo a la norma de la ASTM (1983), sección 4, volumen 04.09, en probetas de madera de $50 \times 50 \times 50 \mathrm{~mm}$, acondicionadas a un $12 \%$ de contenido de humedad. Se tomaron 10 muestras por cada troza (10 trozas en total, lo que nos da un total de 100 muestras). De cada una de las trozas también se prepararon muestras para efectuar cortes al microtomo y maceraciones, con el objeto de efectuar las observaciones y mediciones microscópicas correspondientes. Para la preparación, corte y montaje de las laminillas utilizadas en las observaciones microscópicas se siguieron técnicas conocidas para el estudio de tejidos leñosos (Burger y Richter, 1991). Toda la madera se encontraba libre de plagas y ataques de hongos.

\section{RESULTADOS Y DISCUSION}

\section{Características macroscópicas y} organolépticas. Anillos de crecimiento indistintos o ausentes, duramen de color uniforme, con tonalidades cafés, rojizas, amarillas y blancas, o grises. Color de la albura distinto del color del duramen. Olor indistinto o ausente. Dirección del grano (hilo) recto. Madera lustrosa. Densidad 0.45 - $0.60 \mathrm{~g} / \mathrm{cm}^{3}$. Porosidad difusa, vasos dispuestos en patrón oblicuo y/o radial, generalmente en grupos radiales cortos (2-3 vasos) y de tamaño pequeño. Tílides en los vasos. Otros depósitos se pueden observar en los vasos de duramen. El parénquima axial no es visible con lupa. Radios muy finos, de un solo tamaño. Altura de los radios grandes, normalmente menor de 1 $\mathrm{mm}$.

Características microscópicas. De la tabla 1 y considerando la clasificación empleada para denominar las características microscópicas mensurables presentada por López y Ortega (1989), la madera de Persea americana resultó ser de fibras extremadamente cortas y anchas, con paredes celulares extremadamente gruesas. Sus vasos son de diámetro mediano y moderadamente abundantes. La altura y ancho de sus radios se califican como medianos y en número moderadamente pocos.

Los valores promedio de la tabla 1 comparados con los reportados por Rogel (1982) para la misma especie, difieren principalmente en lo que respecta a longitud de fibra $(1525 \mu \mathrm{m})$ y grosor de pared celular (3 $\mathrm{mm}$ ). Esta diferencia puede ser atribuida a las características de crecimiento propias de los árboles cultivados y utilizados en este estudio, en el que se utilizaron una alta proporción de trozas provenientes de ramas, y por consiguiente con una alta presencia de madera de reacción, que suele generar paredes celulares más gruesas y longitudes de fibra más cortas.

\section{Factibilidad de la madera de aguacate para la elaboración de pulpa. Con relación} a las características morfológicas de las fibras con las propiedades de pulpas elaboradas a partir de esta materia prima, en la tabla 2 se presentan los coeficientes de rigidez, flexibilidad, índice de esbeltez y la relación de Runkel, que permiten visualizar tal comportamiento. 
Tabla 1. Dimensiones de elementos microscópicos de la madera de aguacate

\begin{tabular}{|c|c|c|c|c|c|c|c|}
\hline & \multirow{2}{*}{ Elementos } & \multicolumn{5}{|c|}{ Valor numérico } & \multirow[t]{2}{*}{ Clasificación } \\
\hline & & Mín. & Medio & Máx. & S & C.V. & \\
\hline \multirow{5}{*}{ Fibras } & Longitud, $\mu \mathrm{m}$ & 5696 & 967.7 & 1450.1 & 204.5 & 21.1 & Extrem. corta \\
\hline & Diámetro, $\mu \mathrm{m}$ & 237 & 35.6 & 50.8 & 7.1 & 20.0 & Extrem. ancha \\
\hline & Diámetro de lumen, $\mu \mathrm{m}$ & 118 & 21.3 & 36.9 & 6.8 & 32.1 & Extrem. ancho \\
\hline & Grosor de pared, $\mu \mathrm{m}$ & 45 & 7.2 & 10.7 & 1.42 & 19.9 & Extrem. gruesa \\
\hline & Diámetro, $\mu \mathrm{m}$ & 241 & 81.8 & 145.4 & 29.4 & 35.9 & Mediano \\
\hline \multirow{2}{*}{ Vasos } & Núm. vasos $/ \mathrm{mm}^{2}$ & 80 & 13.1 & 220 & 3.4 & 26.2 & Mod. abundantes \\
\hline & Altura, $\mu \mathrm{m}$ & 1307 & 331.3 & 693.6 & 133 & 403 & Mediana \\
\hline \multirow{2}{*}{ Radios } & Ancho, $\mu \mathrm{m}$ & 228 & 50 & 766 & 136 & 272 & Mediano \\
\hline & Núm. radios/mm lineal & 2 & 5.4 & 8 & 1.26 & 23.4 & Mod. pocos \\
\hline
\end{tabular}

$\mathrm{S}=$ Desviación estándar

C.V. = Coeficiente de variación en \%.

Con base sólo en la relación de Runkel y tomado como referencia la clasificación presentada por Larios (1979), la calidad de pulpas obtenidas con la madera aquí estudiada, se clasifica en la categoría de buena. Sin embargo, analizando en forma individual cada uno del resto de coeficientes, se observa que el coeficiente de rigidez es relativamente bajo, por lo que de acuerdo a Tamolang (1961) y Wangaard (1962), son de esperarse resistencias a la tensión relativamente buenas a regulares, ya que conforme este coeficiente tiende a disminuir, tal propiedad se incrementa. Por su parte, el índice de esbeltez es bajo, lo que permite inferir que su resistencia al rasgado también será baja, ya que existe una relación directa entre ambos. Finalmente, por su coeficiente de flexibilidad (que se ubica en un término medio) es de esperarse también largos de ruptura y resistencia a la tensión relativamente buenos a regulares.

\section{CONCLUSIONES}

Existen grandes cantidades de biomasa generada por las actividades de manejo y mantenimiento en los cultivos de aguacate ( $P$. americana), las cuales deben

Tabla 2. Relaciones morfológicas de la fibra con la calidad de pulpa

\begin{tabular}{||l|c||}
\hline \multicolumn{1}{|c|}{ CRITERIO } & VALOR \\
\hline Relación de Runkel & 0.6738 \\
\hline Coeficiente de rigidez & 0.4029 \\
\hline Índice de esbeltez & 27.1899 \\
\hline Coeficiente de flexibilidad & 0.5979 \\
\hline
\end{tabular}


ser aprovechadas como madera sólida o como materia prima para elaborar pulpa para papel. Sin embargo, debido a las características de crecimiento de los árboles de aguacate cultivados, el mayor volumen de madera aprovechable sería de ramas, por lo que sus propiedades estarían influenciadas por la presencia de madera de reacción.

La madera de aguacate es de color uniforme y puede tener tonalidades amarillas, grises o blancas; es blanda, de textura media a fina, sin veteado distinto. La madera sólida puede ser utilizada para elaborar artesanías y artículos diversos de cortas dimensiones, que no estén sujetos a grandes esfuerzos de resistencia mecánica. En la macroestructura de la madera no se observan caracteres primarios de valor diagnóstico (sensu Silva, 1998) para su identificación, como son un patrón particular de los vasos, visibilidad de parénquima axial, estratificación de elementos y presencia de canales intercelulares.

Con base en las relaciones morfológicas de las fibras de la madera de Persea americana provenientes de huertas, y en especial a la relación de Runkel, la calidad de pulpa resultante se ubica en la categoría de buena en general, pero con baja resistencia al rasgado. En este sentido, su utilización como materia prima para la industria de celulosa y papel es factible de utilizar en mezcla con fibras largas para la manufactura de papeles voluminosos o para fabricación de papel corrugado. Otra opción interesante de estas fibras es para la elaboración a mano de papel para fines artesanales.

\section{REFERENCIAS}

American Society for Testing and Materials. 1983. Designation: D 143-52 (Reapproved 1978) Standard methods for testing small clear specimens of timber. ASTM standards in building codes. Vol. II C170-F656. 25a. Edición. EUA. p: 587-664.

Burger, L.M. y H.G. Richter. 1991. Anatomia da Madeira. Livraria Nobel S. A. Depto. Editorial. Sao Paulo, Brasil. 154 p.

Chudnoff, M. 1984. Tropical timbers of the world. U.S.A. Department of Agriculture. Forest Service. Forest Products Laboratory. Madison, Wisconsin, EUA.

Fuentes T., F.J., J.A. Silva G., y R.M.G. Lomelí. 1998. Indices de hinchamiento y contracción de 3 especies de maderas. Resúmenes de ponencias del II Congreso Mexicano de Tecnología de Productos Forestales. 25-27 Nov. Morelia, Mich. P. 2.

Gama-Campillo, L. 1994. Studies on the systematics and ethnobotany of subgenus Persea. Disertación doctoral. Universidad de California, EUA.

Gama-Campillo, L. 1998. Los aguacates y los antiguos mayas. La Ciencia y el Hombre. Revista de la Universidad Veracruzana 29:83-94.

IAWA Committee. 1989. IAWA List of microscopic features for hardwood identification. IAWA Bull. n.s. 10:219332.

Kopp, L.E. 1966. A taxonomic revision of the genus Persea in the western hemisphere (Persea-Lauraceae). Memoirs of the New York Botanical Garden 14:1-120.

Larios S., P. 1979. Indices de calidad de las pulpas de dos coníferas. Tesis profesional. Departamento de Bosques. Universidad Autónoma de Chapingo. Chapingo, México. 68 p.

López C., H. y F. Ortega E. 1989. Angiospermas arbóreas de México No. 
2. La madera y su uso 23, Boletín técnico. Instituto de Ecología, A.C. Universidad Autónoma MetropolitanaIztapalapa. 128 p.

Martínez-Pinillos C., E. y J. L. Martínez C. 1996. Características de cepillado y lijado de 33 especies de madera. Madera y Bosques 2:11-27.

Pennington, T.D. y J. Sarukhán K. 1998. Arboles tropicales de México. Ediciones Científicas Universitarias. Serie Texto Científico Universitario.Universidad Nacional Autónoma de México y Fondo de Cultura Económica. 521 p.

Quintero, F. 1998. Comunicación personal. Asesor técnico de productores de aguacate y Profesor de la Facultad de Agrobiología de la Universidad Michoacana de San Nicolás de Hidalgo. Michoacán, México.

Record S. J. y R. W. Hess. 1943. Timbers of the New World. Yale University Press, New Haven, Connecticut, EUA. $640 \mathrm{p}$.

Rogel G., Ma. de los A. 1982. Estudio anatómico de la madera de 6 especies tropicales. Bol. Tec. Inst. Nal. Invest. For. 89:37-41.

Silva G., J.A. 1998. Elaboración de una clave analítica para identificación de las maderas que se comercializan en México. Tesis de Maestría en Ciencias de Productos Forestales. Departamento de Madera, Celulosa y Papel "Ing. Karl Augustin Grellmann", Universidad de Guadalajara, Guadalajara, Jalisco, México.

Smith, N.J.H., J.T. Williams, D.L. Plucknett y J.P. Talbot. 1992. Tropical forests and their crops. Comstock Publishing Assoc., Ithaca, New York, EUA. 568 p.

Tamarit U., J.C. 1996. Determinación de los índices de calidad de pulpa para papel de 132 maderas latifoliadas. Madera y Bosques 2:29-41.

Tamolang, F. N. y F. F. Wangaard. 1961. Relationships between hardwood fiber characteristic and pulp-sheet properties. Tappi 44:201-216.

Wangaard, F. F. 1962. Contributions of hardwood fibers to the properties of kraft pulps. Tappi 45:548-556. 
